\title{
Recursos y capacidades: factores que mejoran la capacidad de absorción
}

Resources and capabilities: factors that improve absorption capability

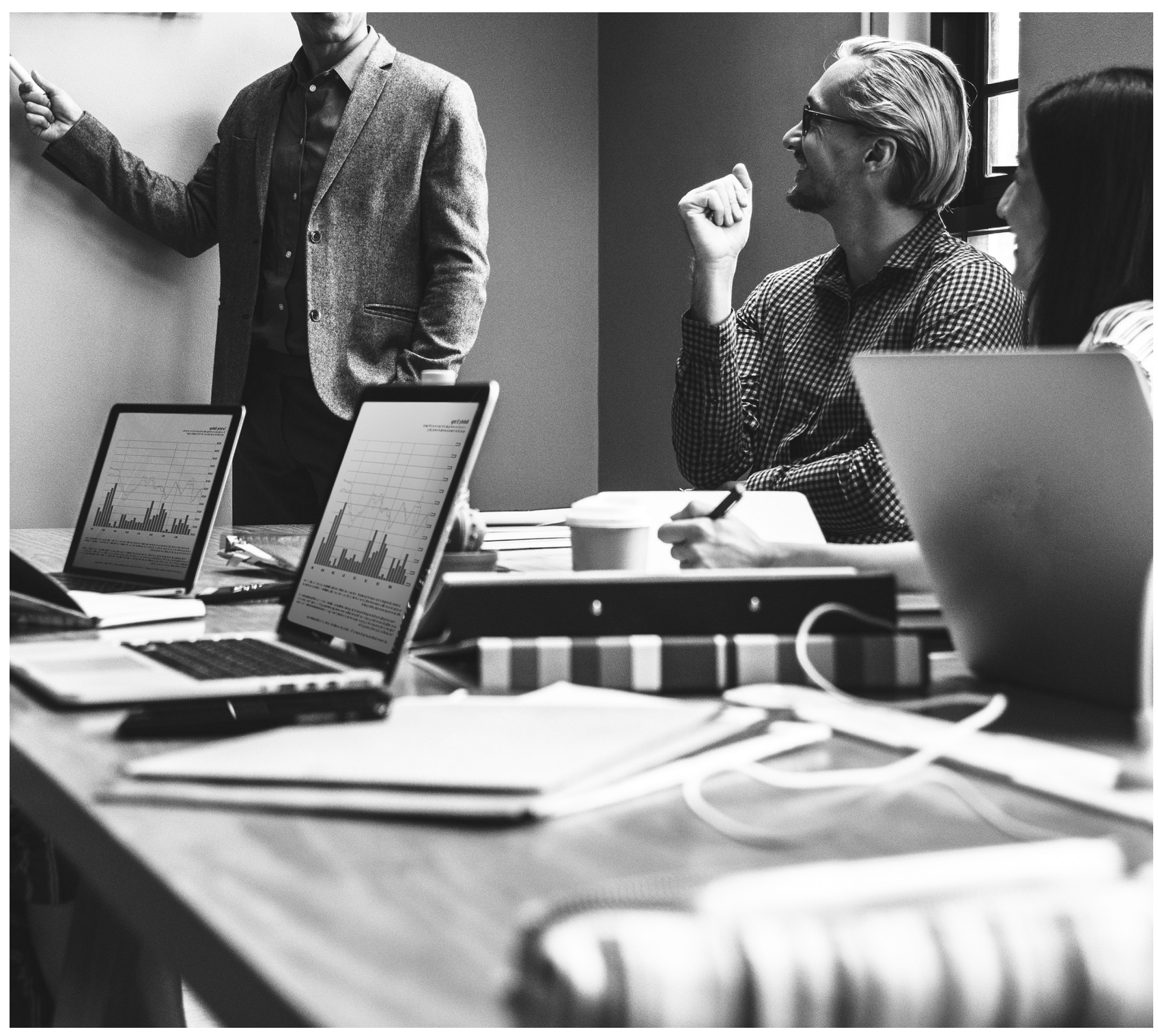




\title{
Recursos y capacidades: factores que mejoran la capacidad de absorción ${ }^{1}$
}

\section{Resources and capabilities: factors that improve absorption capability}

\author{
Mileidy Alvarez-Melgarejo, ${ }^{2}$ Martha Liliana Torres-Barreto $^{3}$
}

Artículo recibido en febrero de 2018; artículo aceptado en marzo de 2018.

Este artículo puede compartirse bajo la Licencia Creative Commons Atribución-No Comercial-Compartir Igual 2.0 Genérica y se referencia usando el siguiente formato: Alvarez-Melgarejo, M. \& Torres-Barreto, M. (2018). Recursos y capacidades: factores que mejoran la capacidad de absorción. I+D Revista de Investigaciones, 12 (2), 51-58.

DOI: https://doi.org/10.33304/revinv.v12n2-2018005

\begin{abstract}
Resumen
Esta investigación se enfoca en el análisis de los recursos tangibles y las capacidades dinámicas de las empresas industriales colombianas de acuerdo con la Teoría de Recursos y Capacidades. Toma como referencia la base de datos EDIT Industria, contrastando empíricamente un modelo mediante regresión lineal múltiple. Los resultados permiten concluir que los recursos financieros influyen positivamente sobre la capacidad de absorción de las empresas industriales colombianas. Asimismo, las capacidades dinámicas de cooperación y de relacionarse con el entorno tienen una influencia sobre la capacidad de absorción de las organizaciones.
\end{abstract}

Palabras clave: recursos tangibles, capacidad de absorción, transferencia de conocimiento, cooperación.

\begin{abstract}
This research focuses on analysis of tangible resources and the dynamic capabilities of Colombian industrial companies according to Resource Based View Theory of the firm. It uses the database: EDIT Industry database, and empirically contrast a model by means of multiple linear regression. The results allow to conclude that financial resources have a positive influence on the absorption capacity of companies. Likewise, the dynamic capabilities of cooperation and of relationship of the firm with its environment have an influence on the absorptive capability of firms.
\end{abstract}

Keywords: Tangible resources, absorptive capability, knowledge transfer, cooperation.

\footnotetext{
1. Investigación empírica con enfoque cuantitativo, resultado de un proyecto de investigación que se encuentra en curso, perteneciente al área de Desarrollo Empresarial Competitivo, subárea de Análisis Empresarial, desarrollado en el grupo de investigación Porter y Finance and Management Fue financiado por la Universidad de Investigación y Desarrollo de la ciudad de Bucaramanga (Colombia) y por la Universidad Industrial de Santander.

2. Grupo de Investigación Porter, Universidad de Investigación y Desarrollo de la ciudad de Bucaramanga (Colombia). Dirección: Calle 9 n. ${ }^{\circ} 23$ - 55 , PBX: 6352525. ORCID ID: http://orcid.org/0000-0003-1752-8023. Correo electrónico institucional: malvarez2@udi.edu.co.

3. Grupo de Investigación Finance and Management, Universidad Industrial de Santander de la ciudad de Bucaramanga (Colombia). Dirección: Cra. 27 Calle 9, PBX: 6344000. ORCID ID: http://orcid.org/0000-0002-6409-5593. Correo electrónico institucional: mltorres@uis.edu.co.
} 


\section{Introducción}

El análisis del sector industrial se considera pertinente desde un punto de vista económico, ya que éste representa una parte significativa en la estructura productiva de un país e influye directamente en su Producto Interno Bruto (PIB) (Noriega et al., 2013). Si a lo anterior se añade que el sector industrial es uno de los que se ve más presionado por el dinamismo del mercado, se podría afirmar que esta situación lo obliga a desarrollar más rápidamente estrategias que le permitan competir y adaptarse a los cambios y demandas del entorno (Calderón, Álvarez \& Naranjo, 2009). Es precisamente este hecho uno de los que motiva esta investigación, que pretende demostrar empíricamente si existen relaciones de dependencia entre recursos y capacidades de las empresas del sector industrial colombiano. De forma concreta, se han seleccionado los recursos tangibles (entre ellos los recursos físicos y financieros), y las capacidades dinámicas; en concreto, la capacidad de absorción, que está definida como la habilidad que tiene la empresa para adquirir conocimiento del entorno, asimilarlo, transformarlo y explotarlo (Torres-Barreto, Martínez, Meza-Ariza, \& Molina, 2016; Zahra \& George, 2002).

\section{Metodología}

\section{Tipo de estudio}

Esta investigación toma como referencia una base de datos existente: la Encuesta de Desarrollo e Innovación Tecnológica en el Sector Manufacturero (EDIT Industria), realizada de forma bianual por el Departamento Administrativo Nacional de Estadística [DANE] en Colombia (Torres-Barreto \& Antolinez, 2017), dirigida a empresas industriales con al menos diez empleados. La encuesta EDIT brinda información estadística acerca de la dinámica del cambio técnico y organizacional, que resulta relevante dado que los datos dan a conocer las tendencias relacionadas con la inversión en actividades de desarrollo e innovación tecnológica y su situación con respecto a otros países (DANE, 2017).

El presente estudio analiza una serie histórica que constituye una base de datos con 589 variables aplicada a 10133 empresas, de las cuales se obtuvo información de 8835 (DANE, 2015). El estudio tiene un diseño transversal, en tanto que la información final se obtiene de un único momento, en este caso, del año 2014.

La selección de variables proxies de recursos financieros y capacidades dinámicas de absorción se llevó a cabo con información encontrada en la literatura y la suministrada por la encuesta EDIT. Inicialmente, se obtuvo un grupo de 120 variables, sin embargo, después de un extenso proceso de revisión, fueron seleccionadas aquellas que brindaban un aporte significativo para el propósito de la investigación. Finalmente, después de que la base de datos estuvo refinada, el estudio incluyó 39 variables medidas para 2093 empresas industriales colombianas.

\section{Variables}

Variable dependiente. La medición empírica de las capacidades dinámicas de absorción se llevó a cabo tomando como referencia investigaciones anteriores encontradas en la literatura y datos de la encuesta EDIT, dado que aún no existe una medida válida y definitiva que incorpore sus diversas dimensiones (Wang \& Ahmed 2007; Flatten, Engelen, Zahra \& Brettel, 2011).

En este sentido, la mayoría de los estudios con métodos cuantitativos han utilizado proxies de esta capacidad (McKelvie \& Davidsson, 2009; Tsai, 2004), centrados en mayor medida en la capacidad de absorción con proxies de I+D (Flatten et al., 2011; Lane, Kokak \& Pathak, 2006). Por su parte, el trabajo de Dutta, Narasimhan y Rajiv (2005) brinda información útil sobre cómo medir capacidades (McKelvie \& Davidsson, 2009). Esta investigación ha utilizado estos trabajos como referencia y, en analogía con las diferentes definiciones encontradas en la literatura (Cohen \& Levinthal, 1990; Lane et al., 2006; Mowery \& Oxley, 1995; Van den Bosch, Van Wijk \& Volberda, 2003; Zahra \& George, 2002), se adopta principalmente el constructor de Zahra y George (2002), quienes consideran que el intercambio de conocimiento es una parte relevante en el proceso de adquirir, asimilar, transformar y explotar el conocimiento.

En este sentido, se analizan y estudian las capacidades dinámicas de absorción, utilizando como proxy la inversión en transferencia de tecnología y adquisición de otros conocimientos. Esta variable representa la identificación y la adquisición del conocimiento técnico o de otro tipo, generado externamente para ser utilizado en innovaciones de la empresa (López, Mejía \& Schmal, 2006), y sostenimiento de la ventaja competitiva (Lache et al., 2016); adicionalmente, está en línea con el argumento de que la capacidad de absorción se refiere a la transferencia de tecnología interna (Lichtenthaler \& Lichtenthaler, 2010).

Variables independientes. Se selecciona un tipo de recurso tangible (recurso de tipo financiero), junto con la capacidad de cooperación y de relacionarse. Los recursos financieros en la EDIT se identifican con las ventas, exportaciones, dinero público y privado invertido en actividades de ciencia, tecnología e innovación, 
provenientes de líneas de financiación, cofinanciación, créditos y recursos propios, que se sustentan en la teoría basada en los recursos. Estas variables representan el dinero propio de la empresa, o dinero externo que ha ingresado y con el que la empresa cuenta (Barney \& Arikan, 2001; Blázquez \& Mondino, 2012; Galbreath, 2005; Huerta, Navas, \& Almodóvar, 2004; Ismail, Rose, Uli, \& Abdullah, 2012; McKelvie \& Davidsson, 2009; Sáez de Viteri Arranz, 2000; Torres-Barreto, 2017; Torres-Barreto, Mendez-Duron, \& Hernandez-Perlines, 2016).

Por otra parte, la capacidad de cooperación y de relación representan un tipo de vínculo de poder que influye en la absorción de nuevos conocimientos (Todorova \& Durisin, 2007); asimismo, Lichtenthaler and Lichtenthaler (2010) exponen que las empresas se relacionan o forman alianzas con el objetivo de transferir tecnología y alcanzar ventajas competitivas (Ariza, 2015).

Variables de control. Se controló el tamaño de la empresa a través de la cantidad de empleados. El $44 \%$ de las empresas incluidas tenían entre 1 y 50 empleados, y el $56 \%$, más de 50 empleados. Finalmente, se utilizaron ocho variables ficticias para la transferencia de tecnología y adquisición de otros conocimientos con el fin de ejercer cierto nivel de control para el sector industrial.

\section{Modelo teórico}

En la Figura 1 se presenta el constructo teórico de la investigación, en el cual se propone que tanto los recursos financieros como las capacidades dinámicas de cooperación y relación con entidades del entorno, inciden sobre la capacidad de la empresa para absorber conocimiento del entorno.

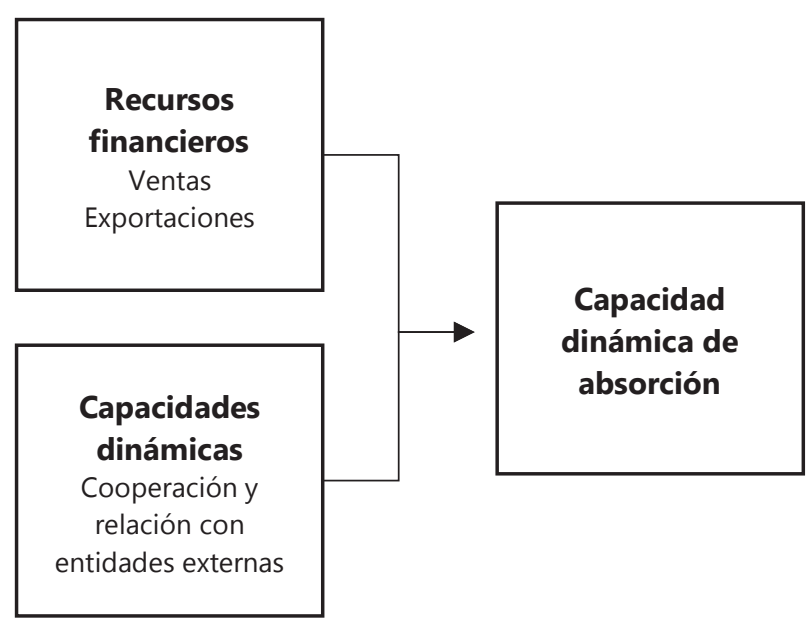

Figura 1. Constructo teórico de la investigación. Fuente: Autores

\section{Resultados}

Con el propósito de probar la existencia de correlaciones entre los recursos tangibles y las capacidades dinámicas, se calculó la matriz de correlación de Pearson. En la Tabla 1 se observa el grado de asociación entre las variables.

Los coeficientes de la correlación se encuentran entre $-0,059$ y 0,701 . De las 12 variables, 9 alcanzaron un nivel de significancia positiva $(p<, 05)$ y solo 3 variables se correlacionaron con un nivel de significancia negativo.

De la matriz de correlaciones se puede observar que las mayores relaciones se presentan entre las variables inversión en transferencia de tecnología y las ventas $(0,701)$, también entre relaciones de las empresas con DNDA y exportaciones $(0,625)$. Otra correlación alta se registra entre las cooperaciones de las empresas con CDT y las cooperaciones con las universidades $(0,522)$, al igual que con las cooperaciones con Parques Tecnológicos $(0,686)$. Estas relaciones son de magnitud elevada y de signo positivo, representando así una asociación fuerte entre la capacidad de absorción y los recursos financieros, los diferentes actores del entorno con que las empresas cooperan y se relacionan.

Para efectuar el análisis de causalidad se recurrió a una técnica de regresión lineal en la que se incluyeron 12 variables independientes articuladas en una regresión lineal múltiple, con coeficientes no estandarizados y errores estándar robustos para la prueba de la hipótesis. La variable dependiente (inversión en transferencia de tecnología y adquisición de otros conocimientos) se transformó en logaritmo natural. Esta transformación se hizo con el fin de lograr que la variable se distribuyera normalmente, homogenizar la base de datos, reducir la heteroscedasticidad y la asimetría, y hacer que las estimaciones fueran más robustas (Gujarati \& Porter, 2009; Mukaka, 2012).

Tal como se observa en la Tabla 2, el modelo es estadísticamente significativo, dado que el $84,82 \%$ de la capacidad de absorción está siendo explicada por los recursos financieros y las capacidades de la empresa para cooperar y relacionarse con entidades del entorno (R2). El cálculo de los factores de inflación de la varianza (VIF) confirmó que el modelo no presenta multicolinealidad.

El test de White indica un p-valor de 0,43; en este sentido, con el $95 \%$ de confianza se tiene evidencia estadística para aceptar la hipótesis nula de homocedasticidad. Se evidencia que las variables independientes y la variable de control tienen un efecto estadísticamente significativo sobre la variable en estudio $(p<, 05)$. 


\section{Discusión de resultados}

Los resultados sugieren que un aumento en las ventas o un aumento en las exportaciones conlleva un incremento de la inversión en transferencia de tecnología y adquisición de conocimientos (a pesar de que las magnitudes son bajas, la relación de causalidad es positiva y significativa). Esto indica que los recursos financieros con los que cuentan las empresas influyen positivamente en su capacidad de absorber información del entorno. En este sentido, cuanto mayor sean las ventas o las exportaciones de las empresas, mayor será su capacidad para absorber conocimiento del medio que la rodea.
Por su parte, las relaciones de las empresas con Icontec y Ministerios, así como el hecho de cooperar con proveedores y CDT, ejercen una influencia positiva sobre la capacidad de absorción. En este sentido, la variable regresora que mayor influencia positiva tiene sobre la regresada es la cooperación de la empresa con CDT $(3,882)$, resultado que sugiere que los CDT están siendo efectivos en su función de desarrollo de proyectos de investigación aplicada, desarrollo de tecnología propia y apoyo a las actividades de transferencia de conocimiento.

Tabla 1

Coeficientes de correlación de Pearson

\begin{tabular}{|c|c|c|c|c|c|c|c|c|c|c|c|c|c|}
\hline & 1 & 2 & 3 & 4 & 5 & 6 & 7 & 8 & 9 & 10 & 11 & 12 & 13 \\
\hline $\begin{array}{l}\text { 1. LNVERSIÓN EN } \\
\text { TRANSFERENCIA DE TECNOLOGÍA } \\
\text { 2. VENTAS }\end{array}$ & 0,701 & 1 & & & & & & & & & & & \\
\hline 3. EXPORTACIONES & 0,216 & 0,201 & 1 & & & & & & & & & & \\
\hline 4. RELACIÓN ICONTEC & 0,132 & 0,084 & 0,230 & 1 & & & & & & & & & \\
\hline 5. RELACIÓN SIC ${ }^{4}$ & $-0,184$ & 0,024 & 0,291 & 0,262 & 1 & & & & & & & & \\
\hline 6. RELACIÓN DNDA ${ }^{5}$ & 0,091 & 0,240 & 0,625 & 0,410 & 0,301 & 1 & & & & & & & \\
\hline 7. RELACIÓN MINISTERIOS & 0,090 & 0,126 & 0,277 & 0,192 & 0,433 & 0,454 & 1 & & & & & & \\
\hline 8. COOPERACIÓN PROVEEDORES & 0,189 & 0,184 & 0,153 & 0,274 & 0,139 & 0,217 & 0,120 & 1 & & & & & \\
\hline 9. COOPERACIÓN CONSULTORES & 0,251 & 0,229 & 0,162 & $-0,016$ & 0,128 & $-0,019$ & 0,236 & 0,300 & 1 & & & & \\
\hline $\begin{array}{l}\text { 10. COOPERACIÓN } \\
\text { UNIVERSIDADES }\end{array}$ & 0,235 & 0,347 & 0,367 & 0,145 & 0,190 & 0,358 & 0,309 & 0,285 & 0,388 & 1 & & & \\
\hline 11. COOPERACIÓN CDT ${ }^{6}$ & 0,135 & 0,137 & 0,252 & 0,194 & 0,135 & 0,429 & 0,236 & 0,198 & 0,339 & 0,522 & 1 & & \\
\hline $\begin{array}{l}\text { 12. COOPERACIÓN PARQUES } \\
\text { TECNOLÓGICOS }\end{array}$ & 0,114 & 0,124 & $-0,076$ & 0,041 & $-0,116$ & $-0,059$ & 0,065 & 0,217 & 0,316 & 0,358 & 0,686 & 1 & \\
\hline 13. TOTAL EMPLEADOS & 0,563 & 0,680 & 0,524 & 0,197 & 0,074 & 0,464 & 0,180 & 0,152 & 0,123 & 0,454 & 0,230 & 0,187 & 1 \\
\hline
\end{tabular}

Fuente: Autores

Tabla 2

Resultados del modelo de regresión lineal múltiple

\begin{tabular}{lccc}
\hline $\begin{array}{c}\text { Inversión en transferencia de tecnología y adquisición } \\
\text { de conocimientos }\end{array}$ & Coef. & $\begin{array}{l}\text { Std. Err. } \\
\text { Robust }\end{array}$ & P-Valor \\
\hline Ventas & $4,63 \mathrm{e}-9$ & $4,00 \mathrm{e}-10$ & 0,000 \\
Exportaciones & $1,13 \mathrm{e}-8$ & $3,19 \mathrm{e}-9$ & 0,001 \\
Relación con Icontec & 1,082 & 0,395 & 0,010 \\
Relación con SIC & $-2,152$ & 0,336 & 0,000 \\
Relación con el DNDA & $-6,275$ & 1,010 & 0,000 \\
Relación con Ministerios & 1,853 & 0,482 & 0,001 \\
Cooperación con proveedores & 1,053 & 0,364 & 0,007
\end{tabular}

4. Superintendencia de Industria y Comercio.

5. Departamento Nacional de Derechos de Autor

6. Centros de Desarrollo Tecnológico 


\begin{tabular}{lrrr} 
Cooperación con consultores & $-1,073$ & 0,393 & 0,010 \\
Cooperación con universidades & $-0,796$ & 0,310 & 0,015 \\
Cooperación con CDT & 3,882 & 0,747 & 0,000 \\
Cooperación con parques tecnológicos & $-2,515$ & 0,751 & 0,002 \\
Total empleados & 0,001 & 0,000 & 0,045 \\
Const & 9,186 & 0,356 & 0,000 \\
\hline No. Observaciones 54 & & & \\
$\mathrm{R}^{2} \quad$ O.8482 & & \\
\hline
\end{tabular}

Fuente: Autores

Por otra parte, la relación de las empresas con la Superintendencia de Industria y Comercio y con el DNDA, así como las cooperaciones de la empresa con consultores, universidades y parques tecnológicos, resultaron factores estadísticamente significativos que influyeron negativamente en la inversión en transferencia de tecnología y adquisición de conocimientos (Gómez, 2014). Entre estas, la variable regresora que más afecta a la capacidad de absorción es la cooperación con DNDA $(-6,2754)$. Esto podría deberse a que este organismo se encarga de diseñar, administrar y ejecutar las políticas gubernamentales en materia de derechos de autor y derechos conexos; en otras palabras, proteger los derechos de los autores de obras literarias y artísticas.

Estas actividades crean barreras para las empresas a la hora de identificar y utilizar el conocimiento de forma precisa, situación conocida como ambigüedad causal. Este término es entendido como un mecanismo de aislamiento para proteger los recursos y capacidades claves de la imitación (González \& Nieto, 2007; Mahoney \& Pandian, 1992; Reed \& Defillippi, 1990); de esta forma impide la transferencia de tecnología entre las empresas (Lin, 2003).

Por otra parte, el efecto negativo de las variables independientes estaría indicando que las empresas que invierten en transferencia de tecnología y adquisición de conocimientos tienden a no recurrir a este tipo de cooperaciones. Otra explicación razonable es que sus efectos ocurren sobre otros aspectos, más no directamente en la variable regresada. Adicionalmente, su efecto negativo está influenciado por otras variables incluidas en el modelo, dado que al revisarlas de manera individual la influencia sobre la variable dependiente es positiva.

A partir de esta investigación se propone seguir ahondando en las relaciones entre recursos y capacidades de las empresas industriales colombianas, dado que el entramado de estas es complejo y demanda un análisis continuo y en mayor profundidad. Este trabajo sirve de base para demostrar la factibilidad de analizar estas relaciones usando técnicas econométricas y estadísticas y ofrece una amplia vía de profundización para seguir ahondando en la temática.

\section{Referencias}

Ariza, H. (2015). Revisitando estrategias de sostenibilidad de las empresas a través de una visión sistémica empresarial. I+ D Revista de Investigaciones, 5(1), 2342. https://doi.org/10.33304/revinv.v05n1-2015002

Barney, J. B., \& Arikan, A. M. (2001). The resource-based view: origins and implications. In Handbook of strategic management (pp. 124-188).

Blázquez, M., \& Mondino, A. (2012). Recursos organizacionales: Concepto, clasificación e indicadores. Instituto de Administración Facultad de Ciencias Económicas Universidad Nacional de Córdoba, 1, V11.

Calderón, G., Álvarez, C., \& Naranjo, J. (2009). Orientación estratégica y recursos competitivos: un estudio en grandes empresas industriales de Colombia. Cuadernos de Administracion, 22(38), 49-72.

Cohen, W., \& Levinthal, D. (1990). Absorptive Capacity: A new perspective on learning and innovation. Adminstrative Science Quarterly, 35(1), 128-152.

Departamento Administrativo Nacional de Estadística. (2015). Encuesta de Desarrollo e Innovación Tecnológica EDIT-Industria-VII. Recuperado de http:// www.dane.gov.co/

Departamento Administrativo Nacional de Estadísticas. (2017). Encuesta de Desarrollo e Innovación Tecnológica (EDIT). Recuperado de http://www.dane.gov.co/index. php/estadisticas-por-tema/tecnologia-e-innovacion/ encuesta-de-desarrollo-e-innovacion-tecnologica-edit

Dutta,S., Narasimhan, O.,\&Rajiv, S. (2005).Conceptualizing and measuring capabilities: Methodology and empirical application. Strategic Management Journal, 26(3), 277-285. https://doi.org/10.1002/smj.442

Flatten, T., Engelen, A., Zahra, S., \& Brettel, M. (2011). A measure of absorptive capacity: Scale development and validation. European Management Journal, 29(2), 98-116. https://doi.org/10.1016/j.emj.2010.11.002

Galbreath, J. (2005). Which resources matter the most to firm success? An exploratory study of resourcebased theory. Technovation, 25, 979-987. https://doi. org/10.1016/j.technovation.2004.02.008

Gómez, F. (2014). Colombia en la inserción de la economía internacional. I+D Revista de Investigaciones, 4(2), 
104-111. https://doi.org/10.33304/revinv.v04n22014009

González, N., \& Nieto, M. (2007). El papel de la ambigüedad causal como variable mediadora entre las prácticas de recursos humanos de alto compromiso y los resultados corporativos. Revista Europea de Dirección y Economía de La Empresa, 16(4), 107-126.

Gujarati, D. N., \& Porter, D. C. (2009). Econometría. McGraw-Hill.

Huerta, P., Navas, J., \& Almodóvar, P. (2004). La Diversificación desde la Teoría de Recursos y Capacidades. Cuadernos de Estudios Empresariales, 14, 87-104.

Ismail, A. I., Rose, R. C., Uli, J., \& Abdullah, H. (2012). The relationship between organisational resources, capabilities, systems and Competitive Advantage. Asian Academy of Management Journal, 17(1), 151-173. Recuperado de https://doi.org/10.3923/ ibm.2012.176.186

Lache, L., León, A. P., Bravo, E., Becerra, L. E., \& Forero, D. (2016). Las tecnologías de información y comunicación como prácticas de referencia en la gestión de conocimiento: una revisión sistemática de la literatura. Revista UIS Ingenierías, 15(1), 27-40.

Lane, P., Koka, B., \& Pathak, S. (2006). The reification of absorptive capacity: A critical review and rejuvenation of the construct. Academy of Management Review, 31(4), 833-863. Recuperado de https://doi.org/10.5465/AMR.2006.22527456

Lichtenthaler, U., \& Lichtenthaler, E. (2010). Technology transfer across organizational boundaries: absorptive capacity and desorptive capacity. California Management Review, 53(1), 154-170.

Lin, B. (2003). Technology transfer as technological learning: a source of competitive advantage for firms with limited $R \& D$ resources. $R \& D$ Management, 33(3), 327-341. Recuperado de https://doi. org/10.1111/1467-9310.00301

López, M., Mejía, J., \& Schmal, R. (2006). Un acercamiento al concepto de la transferencia de tecnología en las universidades y sus diferentes manifestaciones. Panorama Socioeconómico, 24(32), 70-81.

Mahoney, J., \& Pandian, J. (1992). The Resource -Based View Within the Conversation of Strategic Management. Strategic Management Journal, 13(5), 363-380. Recuperado de https://doi.org/10.1002/smj.v13

McKelvie, A., \& Davidsson, P. (2009). From resource base to dynamic capabilities: an investigation of new firms. British Journal of Management, 20(1).

Mowery, D., \& Oxley, J. (1995). Inward technology transfer and competitiveness: the role of national innovation systems. Cambridge Journal of Economics, 19(1), 67-93.

Mukaka, M. (2012). A guide to appropriate use of Correlation coefficient in medical research. Malawi
Medical Journal, 24(3), 69-71. Recuperado de https:// doi.org/10.1016/j.cmpb.2016.01.020

Noriega, J., Gallego, C., López, L., \& Bonilla, A. (2013). Perfil del sector manufacturero Colombiano. Magazín Empresarial, 9(19), 49-61. Recuperado de http://datateca.unad.edu.co/contenidos/102504/ Contenido_curso/2014-II_Contenidos/lectura_ adicional_5._Sector_manufacturero_colombiano.pdf

Reed, R., \& Defillippi, R. (1990). Causal ambiguity, barriers to imitation, and sustainable competitive advantage. Academy of Management Review, 15(1), 88-102.

Sáez de Viteri Arranz, D. (2000). El potencial competitivo de la empresa: Recursos, capacidades, rutinas y procesos de valor añadido. Investigaciones Europeas de Dirección y Economía de La Empresa, 6(3), 71-86. Recuperado de http://www.aedem-virtual.com/ articulos/iedee/v06/063071.pdf

Todorova, G., \& Durisin, B. (2007). Absorptive Capacty: Valuing a Reconceptualization. Academy of Management Review, 32(3), 774-786. Recuperado de https://doi.org/10.5465/AMR.2007.25275513

Torres-Barreto, M. L. (2017). Innovaciones de productos y financiación pública de I+D: Cómo manejar la heterocedasticidad y la autocorrelación. $1+D$ Revista de Investigaciones, 9(1), 138-145. Recuperado de https://doi.org/https://doi.org/10.33304/revinv. v09n1-2017013

Torres-Barreto, M. L., \& Antolinez, D. F. (2017). Exploring the boosting potential of intellectual resources and capabilities on firm's competitiveness. Espacios, 38(31). Recuperado de http://www.revistaespacios. com/a17v38n31/a17v38n31p35.pdf

Torres-Barreto, M. L., Martínez, J. N., Meza-Ariza, L. C., \& Molina, L. P. (2016). El cambio tecnológico en el caso de los textiles inteligentes: Una aproximación desde las capacidades dinámicas. Espacios, 37(8).

Torres-Barreto, M. L., Mendez-Duron, R., \& HernandezPerlines, F. (2016). Technological impact of R\&D grants on utility models. R\&D Management, 46(S2), 537-551.

Tsai, K. (2004). The impact of technological capability on firm performance in Taiwan's electronics industry. Journal of High Technology Management Research, 15(2), 183-195. Recuperado de https://doi. org/10.1016/j.hitech.2004.03.002

Van den Bosch, F., Van Wijk, R., \& Volberda, H. (2003). Absorptive capacity: Antecedents, models and outcomes. ERIM report series research in management. Recuperado de https://doi. org/10.1111/b.9780631226727.2006.00018.x

Wang, C., \& Ahmed, P. (2007). Dynamic capabilities: A review and research agenda. International Journal Management Reviews, 9(1), 31-51. Recuperado de https://doi.org/10.1111/j.1468-2370.2007.00201.x 
Zahra, S., \& George, G. (2002). Absorptive capacity: A review, reconceptualization, and extension. Academy of Management Review, 27(2), 185203. Recuperado de https://doi.org/https://doi. org/10.5465/AMR.2002.6587995 